\title{
Building Virtual Networks Across Multiple Domains
}

\author{
Christoph Werle and \\ Roland Bless \\ Karlsruhe Institute of \\ Technology, Germany \\ \{werle, bless\}@kit.edu
}

\author{
Panagiotis Papadimitriou \\ Leibniz University of \\ Hannover, Germany \\ panagiotis.papadimitriou \\ @ikt.uni-hannover.de
}

Ines Houidi, Wajdi Louati,
and Djamal Zeghlache
Institut Telecom, Telecom
SudParis, France
\{ines.houidi, wajdi.louati,
djamal.zeghlache\}
@it-sudparis.eu

Laurent Mathy

Lancaster University, UK

I.mathy@lancaster.ac.uk

\begin{abstract}
This paper presents a platform for virtual network (VN) provisioning across multiple domains. The platform decomposes VN provisioning into multiple steps to address the implications of limited information disclosure on resource discovery and allocation. A new VN embedding algorithm with simultaneous node and link mapping allows to assign resources within each domain. For inter-domain virtual link setup, we design and realize a signaling protocol that also integrates resource reservations for providing virtual links with Quality-of-Service guarantees. Experimental results show that small VNs can be provisioned within a few seconds.

Categories and Subject Descriptors: C.2.1 [Computer Communication Networks]: Network Architecture and Design
\end{abstract}

General Terms: Design, Management, Performance

Keywords: Network virtualization, resource provisioning, Quality-of-Service, platform design

\section{INTRODUCTION}

Network virtualization has been seen as a promising solution for the concurrent deployment and operation of servicetailored network slices. The wide-area deployment of virtual networks (VNs) creates the need for separation between the network operations and the physical infrastructure. A newly envisioned level of indirection already exists in GENI [3], 4WARD [1], and Cabernet [8]. In this context, VN Providers (VNPs) that act as brokers for virtual resources between VN Operators ${ }^{1}$ (VNOs) and Infrastructure Providers (InPs) will have to provision VNs without having control or even knowledge of any aspect of the physical infrastructure. This entails serious implications on resource discovery and allocation. Existing VN embedding approaches are limited to a single administrative domain, assuming complete knowledge

\footnotetext{
${ }^{1} \mathrm{VN}$ Operators are responsible for the operation of VNs according to the needs of the Service Provider. In some cases VNOs and VNPs (as well as VNOs and Service Providers) may be realized by a single organization.
}

Copyright is held by the author/owner(s).

SIGCOMM'11, August 15-19, 2011, Toronto, Ontario, Canada.

ACM 978-1-4503-0797-0/11/08. of physical resources and the underlying substrate topology. Hence, they cannot be used to provision VNs at large scale, i.e., across multiple domains. Recent work [2] presents an inter-domain VN embedding framework but lacks the required embedding algorithms and a prototype implementation.

In this paper, we present a platform for $\mathrm{VN}$ provisioning across multiple domains that particularly addresses the intricacies of inter-domain aspects. The platform allows VNOs to request VNs from VNPs, which subsequently coordinate their construction by assembling resources from multiple InPs, in line with the VN architecture exemplified in [7]. Since these actors access VN descriptions at different levels of abstraction, we decompose $\mathrm{VN}$ provisioning into a sequence of steps: (i) resource discovery, (ii) resource assignment, and (iii) VN instantiation. We propose and use a new VN embedding algorithm with simultaneous node and link mapping for resource assignment within InPs. To provide the required interoperability for virtual link setup across multiple domains, we design and implement a corresponding signaling protocol.

\section{PLATFORM OVERVIEW}

This section gives an overview of our platform. The platform provides (i) a control plane for $\mathrm{VN}$ provisioning and management, (ii) an XML-based resource and network topology description language, and (iii) on-the-fly VN embedding and instantiation by synthesizing existing technologies, such as Xen for node virtualization and Click Modular Router (running in Linux kernel) for packet encapsulation / decapsulation and packet forwarding. For virtual link setup, we employed IETF's Next Steps in Signaling (NSIS) Framework and extended the QoS NSIS Signaling Layer Protocol (QoS NSLP) to additionally carry required information within a Virtual Link Setup Protocol (VLSP) object $[5,6]$.

Inter-domain VN provisioning is coordinated by VNPs, which have limited resource and topology information of the underlying substrates. Hence, virtual resources should be initially discovered and matched at a rather high level of abstraction (i.e., VNP) to identify candidate resources from which the most appropriate resources will be selected based on detailed information only available to InPs. To this end, our platform provisions VNs as a sequence of the three following steps, which are illustrated in Fig. 1. 


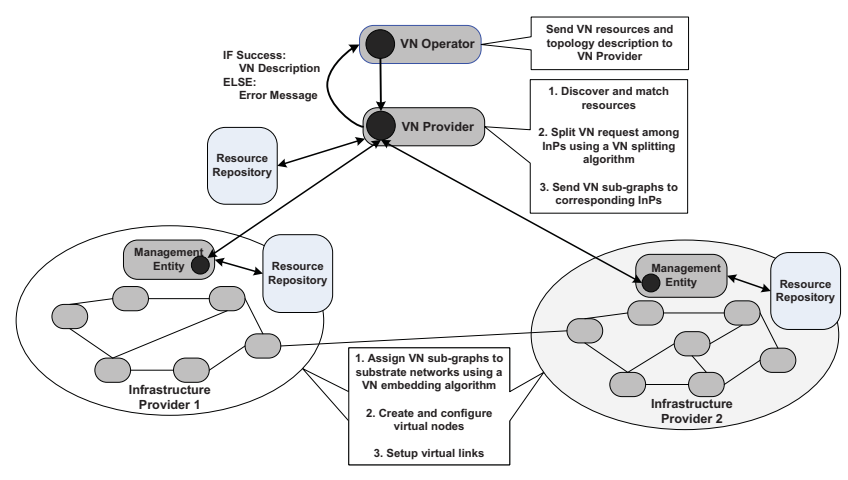

Figure 1: Platform overview with control interfaces and VN provisioning steps.

Resource Discovery: The VNP matches a requested set of virtual nodes and links (which represent a VN topology) to virtual resources offered by InPs. For each virtual node/link instance, the VNP identifies all candidate resources and estimates the cost. Subsequently, the VNP splits the VN topology among the InPs; the objective is to minimize the expenditure for Service Providers. VN partitioning algorithms are discussed in previous work [4].

Resource Assignment: Resource assignment takes place within each InP, since full knowledge of the physical resources and network topology is required. Each InP assigns its partial VN to its substrate network using a VN embedding algorithm. Previous approaches mainly rely on greedy algorithms where node and link mapping is achieved sequentially. Nodes are preselected and assigned, during the node mapping stage, without considering their relation to the link-mapping stage. We use a new heuristic embedding algorithm where node and link mapping phases are simultaneously executed in one stage. The main objective of this algorithm is to optimize load balancing over substrate nodes.

VN Instantiation: Upon resource assignment, the selected substrate resources are allocated by the InPs in order to instantiate the requested VN. VN instantiation is coordinated by a management node within each InP, which signals requests to substrate nodes for virtual node and link setup. We use NSIS to setup virtual links within and between InPs and correspondingly create tunnels with IP-in-IP encapsulation (other tunneling technologies can be used as well). We further discuss virtual link setup in the following section.

\section{VIRTUAL LINK SETUP}

We use the NSIS protocol suite and integrate the virtual link setup with the resource reservation signaling via the QoS NSLP. This integration reduces the setup time of a virtual link, as the resource reservation at the same time conveys the necessary address information of the virtual link. A QoS NSLP extension mechanism for carrying new objects is used to integrate the newly created virtual link setup protocol object (VLSP object). Only the substrate nodes hosting the virtual nodes at the edges of the virtual link need to support the VLSP object and act on it accordingly by, e.g., installing any state required for the setup of the virtual link. Intermediate substrate nodes might be involved in guaranteeing QoS properties of the virtual link or an aggregate of vir-

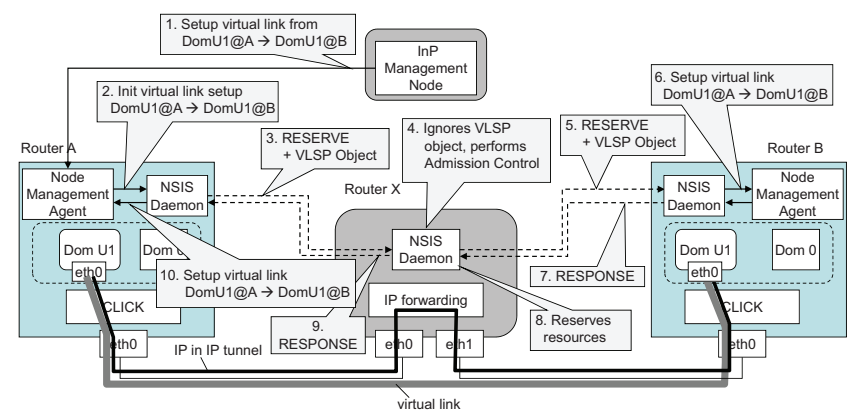

Figure 2: Virtual link setup.

tual links and therefore need to process the remaining QoS NSLP content. They can, however, simply ignore and forward the contained VLSP object, which is ensured by the NSLP object extensions flags in the VLSP object header. Moreover, the path-coupled signaling approach of NSIS assures that a viable substrate path with sufficient resources exists to accommodate the new virtual link.

Fig. 2 illustrates the sequence of events during the setup of a unidirectional virtual link. Experimental results with this setup using a prototypical implementation [6] show that the combined QoS/VLSP signalling does not incur a notable delay during virtual link setup (also if authenticated [5]). Even if the substrate path across two domains comprises several hops, we expect that resource reservation will not dominate the virtual link setup time.

\section{CONCLUSIONS}

We presented a VN platform that complies with the restrictions imposed by multiple domains, such as limited information disclosure. The platform also provides the required interoperability for inter-domain virtual link setup with QoS guarantees.

The platform design shows what technological ingredients are needed and how these can be combined efficiently to provision VNs at large scale. The main building blocks of the platform, such as node virtualization technologies, packet encapsulation/decapsulation and signaling protocols for virtual link setup, are readily available today. This shows that a shift towards full network virtualization is viable in the not too distant future.

\section{REFERENCES}

[1] 4WARD Project, http://www.4ward-project.eu.

[2] M. Chowdhury, F. Samuel, and R. Boutaba, PolyViNE: Policy-based Virtual Network Embedding Across Multiple Domains, Proc. ACM SIGCOMM VISA, New Delhi, India, September 2010.

[3] GENI: Global Environment for Network Innovations, http://www. geni.net.

[4] I. Houidi, W. Louati, W. Bean-Ameur, and D. Zeghlache, Virtual Network Provisioning Across Multiple Substrate Networks, Computer Networks, Vol. 55, No.4, March 2011.

[5] R. Bless, M. Röhricht and C. Werle, Authenticated Setup of Virtual Links with Quality-of-Service Guarantees, Proc. IEEE ICCCN 2011, Hawaii, USA, July 2011.

[6] NSIS-ka, A free C++ implementation of NSIS protocols, KIT, https://svn.tm.kit.edu/trac/NSIS

[7] G. Schaffrath et al., Network Virtualization Architecture: Proposal and Initial Prototype, Proc. ACM SIGCOMM VISA, Barcelona, Spain, August 2009.

[8] Y. Zu, R. Zhang-Shen, S.Rangarajan, and J. Rexford, Cabernet: Connectivity Architecture for Better Network Services, Proc. ACM ReArch '08, Madrid, Spain, December 2008. 